\title{
Study on the Multi-objective Optimal Dispatch of Integrated Community Energy System
}

\author{
Yingrui Wanga ${ }^{\mathrm{a}}$, Jiaqi Shib, ${\text { Tao } \operatorname{Tan}^{\mathrm{c}} \text { and Jianhua Zhang }}^{\mathrm{d}}$ \\ School of Electrical \& Electronic Engineering \\ North China Electric Power University \\ Beijing, China \\ ayingruiw@126.com, b94173546@qq.com, ‘389646508@qq.com, djhzhang001@163.com
}

Keywords: Integrated community energy system, multi-objective, optimal dispatch, operation cost, external pollutant cost.

Abstract. This paper proposes a multi-objective optimization model for the dispatch of the integrated community energy system which is composed of an electricity sub-system, a heat sub-system and a natural gas sub-system. Two objectives, the daily operation cost and external pollutant cost are selected as the objective functions. Mathematical models of the subsystems as well as energy supply devices are given as equality constraints. In addition, inequality constraints that the decision variables must satisfy are also presented. Based on the developed model, the Pareto frontier of the multi-objective optimal dispatch model is obtained using NSGA-II. Numerical cases show the operation differences of devices under different objectives.

\section{Introduction}

With energy and environment issues becoming increasingly prominent, to improve the utilization efficiency of various types of energies has become a key problem to be solved to establish a clean, low-carbon, safe and efficient modern energy system. As important parts of the social energy system, the traditional electricity power system, heat supply system and natural gas supply system are planned and operated independently, which separate the coupling of different kinds of energies and limit the operating flexibility of the energy system. The integrated community energy system (ICES), which will be an important form of the next generation energy system, is composed of the electricity power system, heat supply system, and gas supply system [1]. It realizes the coupling of different energy systems in the transmission, distribution, transfer and consumption parts, and has the characteristics of flexible operation, high efficiency and high utilization of renewable energies. Thus, more and more considerations have been given to it.

At present, most researches pay attention to the optimization of ICES. In [2], the coordinated planning of electricity and natural gas transportation infrastructures is studied using the Benders decomposition. In [3], the model of energy hub is established, and the decentralized algorithm is used to calculate the multi-carrier optimal power flow. In [4], the hierarchical energy management system for the ICES containing electricity heat and gas is proposed, but the heat network constraints are ignored.

In this paper, the mathematical model of the multi-objective optimal dispatch of ICES is proposed. Firstly, the sub-systems which comprise the ICES are described. On this basis, the variables to be optimized are listed. Then, two objectives, operation cost and external pollutant cost are selected to build the optimization model. The equality constraints and inequality constraints which the variables must meet are presented. Last, numerical cases verify the proposed model, and the operation strategy differences of the energy supply devices under different objectives are compared.

\section{Development of the Mathematical Model}

The ICES is composed of the electricity sub-system, heat sub-system and natural gas sub-system in a region. The electricity network transmits the electricity power from the electricity sources to the electricity loads. The heat sub-system consists of the heat sources, supply network, return network 
and heat loads. The heat water with high temperature transmits heat power to the loads, then becomes cool water and return heat sources through the return network. The natural gas sub-system is composed of gas sources, gas network, compressors and gas loads. The compressors are used to maintain the gas pressure to ensure the transmission of natural gas. And the coupling components such as combined heat and power system (CHP), fuel cell (FC) and gas-fired boiler (GB) can realize the conversion among different kinds of energies to meet customers' requirements.

\section{Decision Variables}

The devices in the ICES studied in this paper include the CHP driven by microturbines, FC, electricity energy storage (ES), GB, and ground source heat pump (GSHP). The multi-objective optimal dispatch of the ICES is to find the optimal solution in which all required objective functions are minimized based on their priorities while several equality and inequality constraints are satisfied. The selected decision variables of the problem in this paper are electricity power outputs of CHP, FC, ES, power exchange with the utility grid, heat power outputs of GB, GSHP, and amount of gas bought from the external gas station. Thus the decision variables can be expressed as follows:

$$
\boldsymbol{X}=\left[P_{C H P} ; P_{F C} ; P_{E S}^{c h} ; P_{E S}^{d i s} ; P_{\text {grid }} ; \Phi_{G B} ; \Phi_{G S H P} ; f_{\text {gas }}\right]
$$

\section{Objective Functions}

The operation cost and external pollutant cost of ICES in one day are selected as the objective functions. In the ICES, the fuel consumed by the energy supply devices is natural gas supplied by the gas sub-system. Therefore, the total operation cost includes the startup and shut down cost, the electricity energy exchange cost and the cost to buy natural gas from external gas stations, as shown in (2):

$$
\begin{aligned}
F_{1}(\boldsymbol{X})= & \sum_{t=1}^{T}\left\{\frac{\rho_{e}^{i m}(t)+\rho_{e}^{e x}(t)}{2} P_{\text {grid }}(t)+\frac{\rho_{e}^{i m}(t)-\rho_{e}^{e x}(t)}{2}\left|P_{\text {grid }}(t)\right|\right\} \Delta t \\
& +\sum_{t=1}^{T} \rho_{\text {gas }} f_{\text {gas }}(t) \Delta t+\sum_{t=1}^{T} \sum_{i=1}^{N} S U_{i}\left|u_{i}(\mathrm{t})-u_{i}(t-1)\right|
\end{aligned}
$$

where $S U_{i}$ is the startup and shut down cost of $i$ th device. $u_{i}(t)$ is a binary variable to represent the operation state of $i$ th device, which equals to 1 when it is startup and 0 otherwise. $\rho_{e}^{i m}(t)$ and $\rho_{e}^{e x}(t)$ are the electricity power price of the utility grid. $P_{\text {grid }}(t)$ is the electricity power the ICES exchange with the utility grid. $\rho_{\text {gas }}$ is the gas price, and $f_{\text {gas }}(t)$ is the amount of gas the ICES buys from the external gas stations. $T$ is the total dispatch period, $24 \mathrm{~h} . \Delta t$ is the time interval, $1 \mathrm{~h}$.

In the ICES, the energy supply devices except the photovoltaic (PV) and wind turbine (WT), consume natural gas or electricity, thus they will emit pollutants such as $\mathrm{NO}_{\mathbf{x}} 、 \mathrm{SO}_{2}$ and $\mathrm{CO}_{2}$. The total external pollutant cost includes the pollutant costs of electricity supply devices, heat supply devices and electricity from the utility grid, and can be computed as follows:

$$
F_{2}(\boldsymbol{X})=\sum_{t=1}^{T} \sum_{i=1}^{N_{E}} \sum_{k=1}^{3} \alpha_{k} \lambda_{k, i} P_{i}(t) \Delta t+\sum_{t=1}^{T} \sum_{j=1}^{N_{H}} \sum_{k=1}^{3} \alpha_{k} \lambda_{k, j} \Phi_{j}(t) \Delta t+\sum_{t=1}^{T} \sum_{k=1}^{3} \alpha_{k} \lambda_{\text {grid, }} P_{\text {grid }}^{i m} \Delta t
$$

where $\alpha_{k}$ is the external discount cost of $k$ th pollutant, and $k=1,2,3$ represent $\mathrm{NO}_{\mathrm{x}}, \mathrm{SO}_{2}$ and $\mathrm{CO}_{2}$ respectively. $\lambda_{k, i}$ is the $k$ th pollutant emission factor of $i$ th device. $P_{i}(t)$ is the electricity power of $i$ th electricity supply device, and $\Phi_{\text {grid }}$ is the heat power of $i$ th heat supply device.

\section{Equality Constraints}

The equality constraints can be divided into two parts: network constraints of sub-systems and input-output relationships of devices.

\section{A. Network constraints}

The network constraints are used to represent the distribution of energy flow and power balance. 
At each node of the electricity sub-system, active and reactive power balance must be met, as shown in (4a) and (4b):

$$
\begin{aligned}
& P_{G i}-P_{L i}=V_{i} \sum_{j \in i} V_{j}\left(G_{i j} \cos \theta_{i j}+B_{i j} \sin \theta_{i j}\right) \\
& Q_{G i}-Q_{L i}=V_{i} \sum_{j \in i} V_{i} V_{j}\left(G_{i j} \sin \theta_{i j}-B_{i j} \cos \theta_{i j}\right)
\end{aligned}
$$

where $V_{i}$ is the voltage magnitude of $i$ th node, and $\theta_{i j}$ is the difference of phase angle between $i$ th and $j$ th node. $P_{\mathrm{G} i}, Q_{\mathrm{G} i}$ and $P_{L i}, Q_{L i}$ are the active and reactive power related to the electricity supply devices and loads.

The power balance of the electricity sub-system is shown as follows:

$$
P_{g r i d}(t)+P_{E S}^{d i s}(t)-P_{E S}^{c h}(t)+P_{P V}(t)+P_{W T}(t)+P_{C H P}(t)+P_{F C}(t)=P_{D}(t)+P_{G S H P}(t)+P_{l o s s}(t)
$$

For the heat sub-system, the following equality constraints must be met [5]:

$$
\begin{aligned}
& \Phi_{i}=C_{p} m_{i}\left(T_{s, i}-T_{o, i}\right) \\
& \Phi_{i j}=C_{p} m_{i j}\left(T_{s, \mathrm{i}}-T_{r, i}\right) \\
& T_{j}=\left(T_{i}-T_{a}\right) e^{-\frac{\lambda L}{C_{p} m_{i j}}}+T_{a} \\
& \Phi_{S, i}-\Phi_{L, i}=\sum_{j \in i} \Phi_{i j}
\end{aligned}
$$

Note that (6a) is the heat power expression of node $i$, where $C_{p}$ is the specific heat of water, and $m_{i}$ is the heat water input mass flow. $T_{s, i}$ and $T_{o, i}$ are the supply temperature and output temperature respectively. (6b) is heat power through pipe $i j$, where $m_{i j}$ is the heat water mass flow through pipe $i j$, and $T_{i}, T_{j}$ is the temperature at the start node and end node. $T_{a}$ is the ambient temperature, $\lambda$ is the heat transfer coefficient per unit length and $L$ is the pipe length. $\Phi_{S, I}$ and $\Phi_{L, i}$ are the heat power related to heat supply device and heat load, respectively.

The power balance of the heat sub-system is shown as follows:

$$
\Phi_{\mathrm{GB}}(t)+\Phi_{\mathrm{CHP}}(t)+\Phi_{\mathrm{GSHP}}=\Phi_{\mathrm{D}}(t)+\Phi_{\text {loss }}(t)
$$

where $\Phi_{\text {loss }}(t)$ is heat power loss.

For the natural gas sub-system, the following equality constraints must be satisfied [6]:

$$
\begin{aligned}
& f_{i j}=K_{i j} s_{i j} \sqrt{s_{i j}\left(p_{i}^{2}-p_{j}^{2}\right)} \\
& f_{S i}-f_{L i}=\sum_{j \in i} f_{i j}
\end{aligned}
$$

Note that (8a) is the expression of gas flow through pipe $i j$, where $K_{i j}$ is the pipe coefficient, and $p_{i}$ is gas pressure at node $i$. $s_{i j}$ specifies the direction of gas flow. $s_{i j}=1$ when $p_{i}>p_{j}$ and $s_{i j}=-1$ otherwise. $(8 \mathrm{~b})$ is the flow balance at node $i . f_{S i}$ and $f_{L i}$ are gas flow related to the gas source and load respectively.

The gas flow balance of the natural gas sub-system is shown as follows:

$$
f_{\text {gas }}(t)=f_{D}(t)+f_{C H P}(t)+f_{G B}(t)+f_{F C}(t)+f_{c p}
$$

where $f_{C H P}, f_{G B}, f_{F C}$ and $f_{c p}$ are the gas flow consumed by CHP, GB, FC and the compressor, respectively.

B. Input-output relationships of devices 
The CHP consumes natural gas to generate electricity and heat power. The mathematical mode is shown in (10):

$$
\left\{\begin{array}{l}
f_{C H P}=\frac{1}{q_{g a s}} \frac{P_{C H P}}{\eta_{M T}} \\
\Phi_{M T}=\frac{P_{M T}\left(1-\eta_{M T}-\eta_{l}\right)}{\eta_{M T}} \\
\Phi_{C H P}=\Phi_{M T} \eta_{r e c} \cdot C O P_{h}
\end{array}\right.
$$

where $q_{g a s}$ is the calorific value of natural gas, $\eta_{M T}$ is the electricity generation efficiency of CHP, $\eta_{l}$ is the residual heat loss efficiency, $\eta_{r e c}$ is the residual heat recovering efficiency, and $C O P_{h}$ is the heat production coefficient.

The relationship between natural gas consumption and electricity power of FC is expressed by:

$$
f_{F C}=\frac{P_{F C}}{q_{g a s} \eta_{F C}}
$$

The energy stored in ES $E_{\mathrm{ES}}(t)$ is decided by charge power $P_{E S}^{c h}(t)$ and discharge power $P_{E S}^{d i s}(t)$ :

$$
E_{E S}(t)=E_{E S}(t-1)+\left(\eta_{c h} P_{E S}^{c h}(t)-\frac{1}{\eta_{d i s}} P_{E S}^{d i s}(t)\right) \Delta t
$$

where $\eta_{c h}$ and $\eta_{\text {dis }}$ are the charge and discharge efficiency respectively.

The GSHP is a new type of environment-friendly heat supply device, which consumes electricity to transfer heat under the ground to heat loads. The heat power transferred $\Phi_{\mathrm{GSHP}}$ can be 4 to 5 times of the electricity power consumed $P_{\mathrm{GSHP}}$, so the heat supply cost is very low. In this paper, a simplified model is used, which expresses the relationship between input and output using a quadratic function:

$$
P_{G S H P}=a+b \Phi_{G S H P}+c \Phi_{G S H P}^{2}
$$

The GB burns natural gas to supply heat, and its mathematical model is as follows:

$$
f_{G B}=\frac{1}{q_{g a s}} \Phi_{G B} \cdot C O P_{h}
$$

In the natural gas sub-system, compressors are installed at some pipes. The gas-fired compressor consumes some amount of natural gas when operating, and is expressed as follows:

$$
f_{c p}=\frac{k_{c p} f_{c o m} T_{g a s}}{q_{g a s}}\left(\mathrm{k}_{c p}^{\frac{a-1}{a}}-1\right)
$$

where $k_{c p}$ is the compression ratio, $T_{\text {gas }}$ is natural gas temperature, $f_{c o m}$ is the gas flow through the compressor, and $a$ is the variable index of the compressor.

\section{Inequality Constraints}

To ensure the security operation of the ICES, the state variables $\boldsymbol{x}=[\boldsymbol{V} ; \boldsymbol{\theta} ; \boldsymbol{T} ; \boldsymbol{p}]$ must be within the allowable limits:

$$
\boldsymbol{x}_{\min } \leq \boldsymbol{x} \leq \boldsymbol{x}_{\max }
$$

In addition, energy supply devices should meet the following requirements: 


$$
\begin{aligned}
& u_{i}(t) P_{i, \text { min }}(t) \leq P_{i}(t) \leq u_{i}(t) P_{i, \text { max }}(t) \\
& u_{i}(t) \Phi_{i, \text { min }}(t) \leq \Phi_{i}(t) \leq u_{i}(t) \Phi_{i, \text { max }}(t) \\
& -r_{i, \text { down }} \Delta t \leq P_{i}(t)-P_{i}(t-1) \leq r_{i, u p} \Delta t \\
& -r_{i, \text { down }} \Delta t \leq \Phi_{i}(t)-\Phi_{i}(t-1) \leq r_{i, u p} \Delta t
\end{aligned}
$$

It is worthwhile to note that (16a) and (16b) are the output limits; (16c) and (16d) are the ramp rate limits, where $r_{i, u p}$ and $r_{i, \text { down }}$ are ramp up and ramp down rates respectively.

The electricity power exchange between ICES and the utility grid and natural gas bought from external gas stations must be with in allowable limits, as in (17):

$$
\begin{gathered}
P_{g r i d, \text { min }} \leq P_{\text {grid }} \leq P_{\text {grid, } \text { max }} \\
f_{\text {gas,min }} \leq f_{\text {gas }} \leq f_{\text {gas, max }}
\end{gathered}
$$

\section{Solving the Multi-objective Optimization Problem}

The solution of the multi-objective optimal dispatch problem is a Pareto optimal solution set in which the individuals are the best compromise or trade-off between the two objective functions. Therefore, the fast and elitist multi-objective genetic algorithm NSGA-II is used to solve the problem.

\section{Case Study}

\section{Test System under Study}

The ICES in Fig. 1 is used to study the multi-objective optimal dispatch problem. The electricity sub-system is connected to the utility grid through EB12, the capacity of the PV connected to EB3 and EB8 is $200 \mathrm{~kW}$, the capacity of WT is $1000 \mathrm{~kW}$, and the CHP operates in follow-electricity-load mode. Other parameters are shown in Table 1. The pollutant emission indexes are from [7],the time-of-use electricity price is from [8], and natural gas price is $2.51 \mathrm{CNY} / \mathrm{m}^{3}$. The daily power curves of the PV, WT, electricity load and heat load are shown in Fig.2. The type of the overhead lines is LJ95.

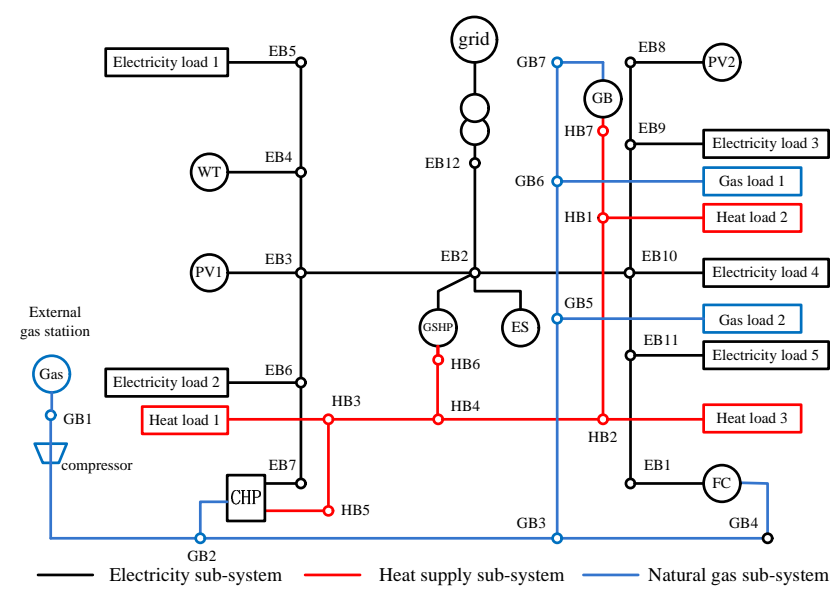

Fig.1 ICES under study

Table 1 Coefficients of energy supply devices in ICES

\begin{tabular}{ccc}
\hline Devices & Minimum power $[\mathrm{kW}]$ & Maximum power $[\mathrm{kW}]$ \\
\hline CHP & 5 & 130 \\
FC & 10 & 200 \\
GSHP & 0 & 200 \\
GB & 100 & 1500 \\
ES & -200 & 200 \\
grid & -600 & 600 \\
\hline
\end{tabular}




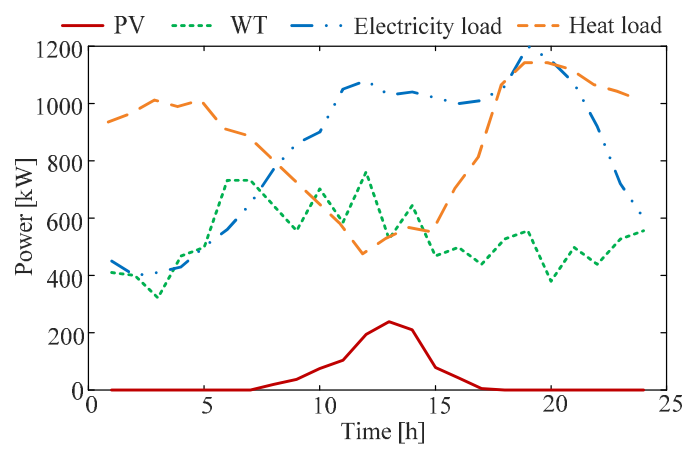

Fig.2 Daily power curve of PV, WT, electricity load and heat load

\section{Simulation Results and Analysis}

The Pareto frontier of the multi-objective optimal dispatch problem is shown in Fig.3. It can be seen that there is a non-linear relationship between the operation cost and pollutant external cost. From the minimum operation cost point (point A) to the minimum pollutant external cost (point B), the pollutant external cost decreases $301 \mathrm{CNY}$, but the operation cost increases $1247 \mathrm{CNY}$.

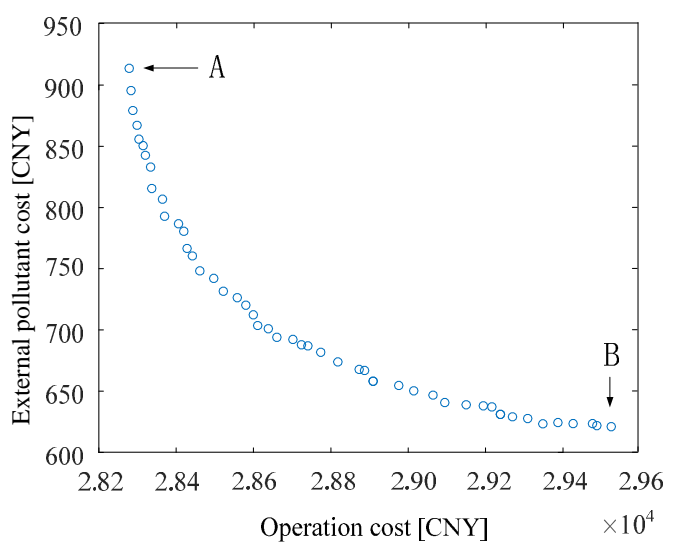

Fig.3 Pareto frontier of ICES multi-objective optimal dispatch

To analyze the operation difference of the ICES under different objectives, the simulation results of point A and point B are listed in Fig.4 and Fig.5.
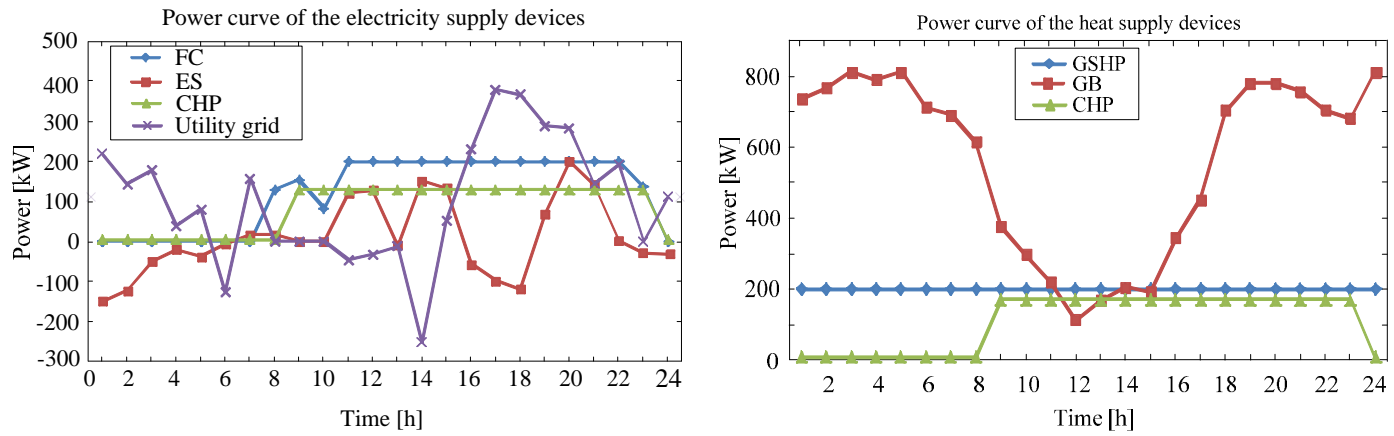

Fig.4 Result of ICES optimal dispatch for point A
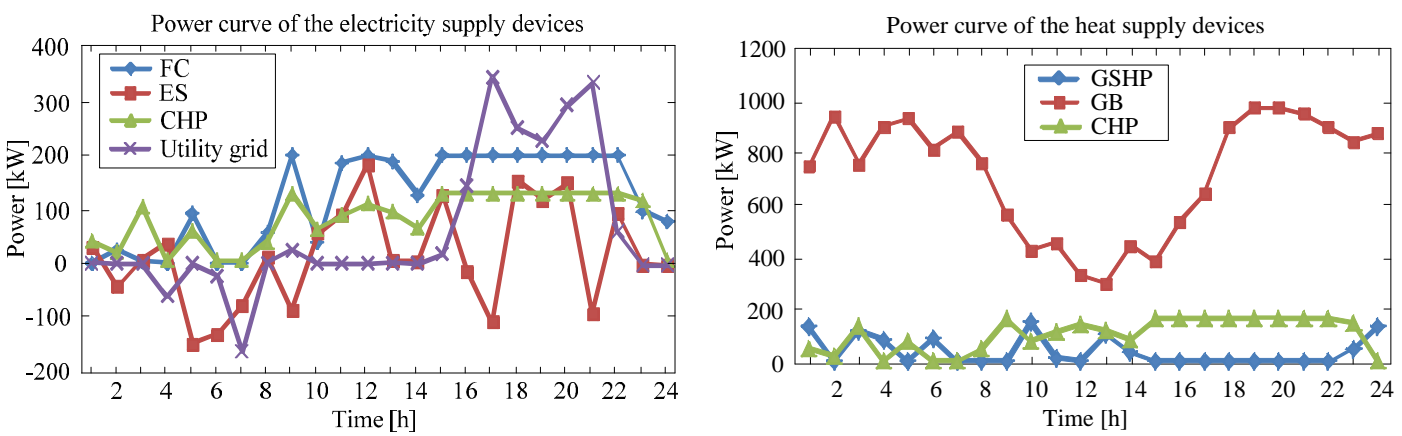

Fig.5 Result of ICES optimal dispatch for point B 
According to Fig.2, at around 6:00 am, the total power of PV and WT exceeds the electricity load, so as shown in Fig.4, ICES exports additional electricity power to the utility grid, as in Fig.4 (a). When electricity price is low, the ES is charged, and when the price is high, it is discharged. The average energy supply cost of CHP is below the high and medium electricity price, so in the relative time intervals the CHP works at its maximum power. The heat supply cost of GSHP is only 0.83/4.5=0.184 CNY when at peak electricity price, so it works the whole day, as in Fig.4 (b).

The amount of pollutant emitted by GSHP (because it consumes electricity) is related to the proportion of electricity from different sources. As in Fig.5, when the ICES imports more electricity from the utility grid, the electricity is dirtier, so at these time intervals the GSHP is shut down. From Fig.5 it can be seen that when ICES operates under the objective of minimum pollutant external cost, the GSHP only works when the ICES export electricity to or import little from the utility grid. The pollutant emitted by GS is lower compared with CHP and GSHP, so it generates much more heat.

It is obvious that energy supply devices which use natural gas as fuel are much cleaner and low-cost, so even if under different operation objectives, they operate most of the time.

\section{Conclusion}

In this paper, a novel multi-objective optimal dispatch model of ICES is proposed to reduce the daily operation cost and external pollutant cost. The sub-system network models and relationships between input and output of energy supply devices are given as equality constraints, and other constraints that the decision variables must satisfy to ensure the security operation of ICES are given as inequality constraints. The operation differences of devices under different objectives are compared by numerical cases. It is obvious that the operation cost and external pollutant cost of devices using natural gas as fuel are much lower than other devices, thus they undertake much of the energy supply. In the future work, uncertainty of PV, WT and loads will be considered in the optimal dispatch problem to increase the dispatch accuracy of ICES.

\section{References}

[1] D. Cartes, J. Ordonez, J. Harrington, et al. Novel Integrated Energy Systems and control methods with economic analysis for integrated community based energy systems[C]/Power Engineering Society General Meeting, 2007. IEEE. IEEE, 2007: 1-6.

[2] X. Zhang, M. Shahidehpour, A. S. Alabdulwahab, et al. Security-constrained Co-optimization planning of electricity and natural gas transportation infrastructures. Power Systems, IEEE Transactions on, Vol. 30 (2015), p. 2984-2993.

[3] M. Arnold, R. R. Negenborn, G. Andersson and B. De Schutter "Distributed control applied to combined electricity and natural gas infrastructures". Infrastructure Systems and Services: Building Networks for a Brighter Future (INFRA), 2008 First International Conference on, Rotterdam, 2008, p. 1-6.

[4] X. Xu, X. Jin, H. Jia, X. Yu and K. Li. Hierarchical management for integrated community energy systems. Applied Energy, Vol. 120 (2015), p. 231-243.

[5] X. Liu. Combined analysis of electricity and heat networks. Cardiff University (2013).

[6] M. Jiang, Y. Xu, S. Wang, et al. Simulation and analysis of gas transmission and distribution network. Beijing: Petroleum Industry Press (1995).

[7] L. Li. Study of economic operation in microgrid. Beijing: North China Electric Power University (2011).

[8] X. Wu, X. Wang, J. Wang, et al. Economic generation scheduling of a microgrid using mixed integer programming. Proceedings of the CSEE, Vol. 33 (2013), p. 1-8. 